\title{
miR-203 affects esophageal cancer cell proliferation, apoptosis and invasion by targeting MAP3K1
}

\author{
MINGZHU ZONG, WANTING FENG, LI WAN, XIAOJUAN YU and WEIYONG YU \\ Department of Oncology, The Affiliated Huaian No. 1 People's Hospital of \\ Nanjing Medical University, Huai'an, Jiangsu 223300, P.R. China
}

Received July 30, 2019; Accepted January 24, 2020

DOI: $10.3892 / \mathrm{ol} .2020 .11610$

\begin{abstract}
R-203 has been indicated to be a tumor suppressor in esophageal cancer, however, the underlying molecular mechanisms by which it functions are not fully understood. The present study aimed to investigate the molecular mechanisms underlying the regulatory activities of microRNA (miR)-203 in esophageal cancer. The miR-203 mimic/inhibitor, Mitogen-Activated Protein Kinase Kinase Kinase 1 (MAP3K1) overexpression plasmid and MAP3K1 small interfering (si)RNA were transfected into TE-1 cells. miR-203 and MAP3K1 mRNA expression were detected via reverse transcription-quantitative PCR analysis, while MAP3K1 protein expression was detected via western blot analysis. Dual-luciferase reporter assay was used to determine whether MAP3K1 was a direct target of miR-203. Cell proliferation and invasion abilities were assessed via MTT and Matrigel assays, respectively. Cell apoptosis was analyzed via flow cytometry, Caspase 8/3 Assay kits and western blot analysis. The results demonstrated that MAP3K1 was a direct target of miR-203. Overexpression of MAP3K1 reversed the suppressed cell proliferation and invasion abilities induced by miR-203 mimic, as well as the inhibitory effect of miR-203 mimic on cell apoptosis. Furthermore, MAP3K1 siRNA weakened the effect of miR-203 inhibitor on cell proliferation, apoptosis and invasion.
\end{abstract}

\section{Introduction}

Esophageal cancer is the sixth most common cause of cancer-associated mortality worldwide in 2013, with an incidence rate of $1 / 100,000$ in China (1). The majority of patients with esophageal cancer are diagnosed in the progressive stage (2). Current treatments available for esophageal cancer result in poor prognosis (3), with a 5-year survival rate of

Correspondence to: Dr Weiyong Yu, Department of Oncology, The Affiliated Huaian No. 1 People's Hospital of Nanjing Medical University, 6 Beijing Road West, Huai'an, Jiangsu 223300, P.R. China E-mail: doctorywy@163.com

Key words: esophageal cancer, microRNA-203, MAP3K1
$13 \%$ (4). Thus, development of novel therapeutic targets for the treatment of esophageal cancer remains critical, and research into the molecular mechanisms underlying tumorigenesis are required.

microRNAs (miRNAs) are small endogenous non-coding RNAs that play essential roles in several complex physiological processes, including cell proliferation and apoptosis, growth and development, organogenesis, immune defense and inflammation $(5,6)$. Aberrant expression of miRNAs has been implicated in a number of human diseases, including cancer (7-9). Some miRNAs were reported to be associated with the origin and development of esophageal cancer, such as miR-21, miR-375 and the miR-17-92 cluster (10). miR-203 functions as a tumor suppressor in several malignancies, and its expression has been reported to vary across different types of tumor (11-14). It has been demonstrated that miR-203 is significantly downregulated in esophageal cancer (15). A previous study reported that overexpression of miR-203 notably induces cell apoptosis, inhibits cell proliferation, migration and invasion in vitro, and suppresses tumor growth in vivo in esophageal cancer cells (16). However, to the best of our knowledge, the molecular mechanisms underlying the regulatory activities of miR-203 in esophageal cancer are not yet fully understood.

The present study aimed to determine whether Mitogen-Activated Protein Kinase Kinase Kinase 1 (MAP3K1) is a direct target of miR-203, and investigate the molecular mechanism underlying the role of miR-203 in esophageal cancer.

\section{Materials and methods}

Cell culture and transfection. TE-1 cells and 293 cells were purchased from The Cell Bank of Type Culture Collection of the Chinese Academy of Sciences, and cultured in RPMI-1640 medium (Gibco; Thermo Fisher Scientific, Inc.) supplemented with $10 \%$ FBS (Gibco; Thermo Fisher Scientific, Inc.), at $37^{\circ} \mathrm{C}$ in a humidified atmosphere of $5 \% \mathrm{CO}_{2}$. Prior to transfection, cells (1x10\%/well) were washed 3 times with PBS and added to serum-free RPMI-1640 medium. A total of $2 \mu \mathrm{g}$ of miR-203 mimic/inhibitor, MAP3K1 pcDNA3.1 and MAP3K1 siRNA were transfected into TE-1 cells using Lipofectamine ${ }^{\mathrm{TM}} 2000$ (Invitrogen; Thermo Fisher Scientific, Inc.), according to the manufacturer's protocol. After $6 \mathrm{~h}$, the medium was replaced with fresh medium and maintained in the cultures for at least $24 \mathrm{~h}$ for further analysis. 
Luciferase reporter assay. The 3'-untranslated region (UTR) segment of MAP3K1 and its mutant were amplified and inserted into the pGL3-control luciferase reporter vector (Promega Corporation). Cells transfected with wild-type or mutated MAP3K1 3'UTR were divided into two groups: Control group and mimic group. Cells in the control group were transfected with the miR-negative control. Reporter plasmids containing 3'UTR MAP3K1 were co-transfected with the miR-203 mimic into 293 cells using Lipofectamine ${ }^{\mathrm{TM}}$ 2000 reagent. $48 \mathrm{~h}$ following transfection, luciferase activity was determined using the Luciferase Reporter Assay System and Luciferase Assay kit (both from Promega Corporation). Results were expressed as the Firefly luciferase activity normalized to Renilla luciferase activity.

Western blotting. In order to examine the effect of miR-203 mimic/inhibitor on MAP3K1 protein expression, cells were divided into three groups: Control group, mimic group and inhibitor group. Cells in the control group were transfected with the miR-negative control. Cells were divided into control, MAP3K1 pcDNA3.1 and MAP3K1 siRNA groups to detect MAP3K1 andFas protein expression in MAP3K1-overexpressed or MAP3K1-knockdown cells. Cells in the control group were transfected with the empty vector + scrambled siRNA. Cells were divided into control, mimic and mimic + MAP3K1 groups to examine whether MAP3K1 could reverse the effect of miR-203 mimic on MAP3K1 and Fas protein expression. Cells in the control group were transfected with the miR-negative control + empty vector. Cells were divided into control, inhibitor and inhibitor + MAP3K1 siRNA groups to determine whether MAP3K1 could reverse the effect of miR-203 inhibitor on MAP3K1 and Fas protein expression. Cells in the control group were transfected with the miR-negative control + scramble siRNA.

Total protein was extracted from cells using ice-cold lysis buffer [150 mM NaCl, $150 \mathrm{mM}$ Tris-HCl (pH 7.4), 0.2\% SDS, $1 \%$ Nonidet P-40, $50 \mathrm{mM}$ sodium fluoride, $100 \mathrm{mM}$ sodium vanadate and $1 \mathrm{mM}$ phenylmethylsulfonyl fluoride]. Protein concentrations were determined using the BCA method. A total of $50 \mu \mathrm{g}$ of protein/lane was separated via SDS-PAGE on a $12 \%$ gel and subsequently transferred onto a nitrocellulose membrane (EMD Millipore). The membranes were blocked with 3\% BSA (Sigma-Aldrich; Merck KGaA) overnight at $4{ }^{\circ} \mathrm{C}$, prior to being washed three times with TBST. Subsequently, the membranes were incubated with primary antibodies against MAP3K1 (dilution, 1:800; cat. no. sc-449), Fas (dilution, 1:400; cat. no. sc-74540;) and GAPDH (dilution, 1:1,000; cat. no. sc-51907) (all from Santa Cruz Biotechnology, Inc.) at $37^{\circ} \mathrm{C}$ for $1 \mathrm{~h}$. Membranes were washed with TBST 3 times and subsequently incubated with horseradish peroxidase-labeled secondary antibodies (dilution, 1:1,000; cat. no. sc-2005; Santa Cruz Biotechnology, Inc.) at $37^{\circ} \mathrm{C}$ for $1 \mathrm{~h}$. Protein bands were visualized using an ECL detection kit (Pierce; Thermo Fisher Scientific, Inc.).

Reverse transcription-quantitative (RT-q)PCR. In order to examine the effect of miR-203 mimic/inhibitor on miR-203 and MAP3K1 mRNA expression, cells were divided into the following three groups: Control group, mimic group and inhibitor group; control, mimic and mimic + MAP3K1 groups, and control, inhibitor and inhibitor + MAP3K1 siRNA groups, as previously described. miRNAs were isolated using the miRNeasy Mini kit (Qiagen $\mathrm{GmbH}$ ), according to the manufacturer's protocol. Total RNA was extracted from TE-1 cells using TRIzol ${ }^{\circledR}$ reagent (Invitrogen; Thermo Fisher Scientific, Inc.). A total of $50 \mathrm{ng}$ of total RNA was reverse transcribed into cDNA using the First Strand cDNA Synthesis kit (Fermentas; Thermo Fisher Scientific, Inc.). qPCR was subsequently performed using the SYBR-Green PCR kit, in a 7900 Sequence Detection System (both from Applied Biosystems; Thermo Fisher Scientific, Inc.) according to the manufacturer's protocols. Thermocycling conditions were as follows: Initial denaturation at $95^{\circ} \mathrm{C}$ for $3 \mathrm{~min}$, followed by 40 cycles of denaturation at $95^{\circ} \mathrm{C}$ for $30 \mathrm{sec}$, annealing at $60^{\circ} \mathrm{C}$ for $30 \mathrm{sec}$ and elongation at $72^{\circ} \mathrm{C}$ for $45 \mathrm{sec}$, final extension at $72^{\circ} \mathrm{C}$ for $5 \mathrm{~min}$. The primers used were: miR-203 forward, $5^{\prime}$-agtggttcttaacagttcaacagtt-3' and reverse 5'-tggtgtcgtggagtcg-3'; U6 forward, 5'-ctcgettcggcagcaca-3' and reverse, 5'-aacgcttcacgaatttgcgt-3'; MAP3K1 forward, 5'-ccacagaga acagttccct-3' and reverse, 5'-ccattggctttggttgctct-3'; GAPDH, forward, 5'-ctgacttcaacagcgacacc-3' and reverse, 5'-gtggtc caggggtcttactc- 3 '. Relative expression levels were measured using the $2^{-\Delta \Delta C q}$ method (17). miRNA levels were normalized to the internal control U6, while MAP3K1 mRNA levels was normalized to the internal reference gene GAPDH.

MTT assay. In order to examine the proliferation of MAP3K1-overexpressed or MAP3K1-knockdown cells, cells were divided into the respective groups: Control group, MAP3K1 pcDNA3.1 group and MAP3K1 siRNA group; control, mimic and mimic + MAP3K1 groups, and control, inhibitor and inhibitor + MAP3K1 siRNA groups, as aforementioned. Cell proliferation was determined via an MTT assay. Cells (1x10 $/$ well) were seeded into 96 -well plates and allowed to grow for the appropriate times $(24,48,72$ and $96 \mathrm{~h})$ at $37^{\circ} \mathrm{C}$ with $5 \% \mathrm{CO}_{2}$, prior to incubation with $10 \mu \mathrm{l}$ of MTT (Sigma-Aldrich; Merck KGaA) at $37^{\circ} \mathrm{C}$ for $4 \mathrm{~h}$. Following MTT incubation, the purple formazan crystals were dissolved using $200 \mu 1$ of dimethyl sulfoxide (Sigma-Aldrich; Merck $\mathrm{KGaA}$ ) and cell proliferation was subsequently analyzed at a wavelength of $570 \mathrm{~nm}$, using a microplate reader (Molecular Devices, LLC).

Flow cytometry analysis of apoptosis. In order to detect the apoptotic rate of MAP3K1-overexpressed or MAP3K1-knockdown cells, cells were divided into the respective groups: Control group, MAP3K1 pcDNA3.1 group and MAP3K1 siRNA group; control, mimic and mimic + MAP3K1 groups, and control, inhibitor and inhibitor + MAP3K1 siRNA groups, as aforementioned. TE-1 cells were washed three times with PBS and resuspended in binding buffer (Nanjing KeyGen Biotech Co., Ltd.). The cells were subsequently stained with $5 \mu \mathrm{l}$ Annexin-V and $5 \mu \mathrm{l}$ propidium iodide (Nanjing KeyGen Biotech Co., Ltd.) for $20 \mathrm{~min}$ at room temperature in the dark. Apoptotic cells were subsequently analyzed using a FACSCalibur flow cytometer (BD Biosciences) and CellQuest Pro software (version 5.2; BD Biosciences).

Caspase $8 / 3$ activity assay. In order to examine caspase 8/3 activity in MAP3K1-overexpressed or MAP3K1-knockdown 
A

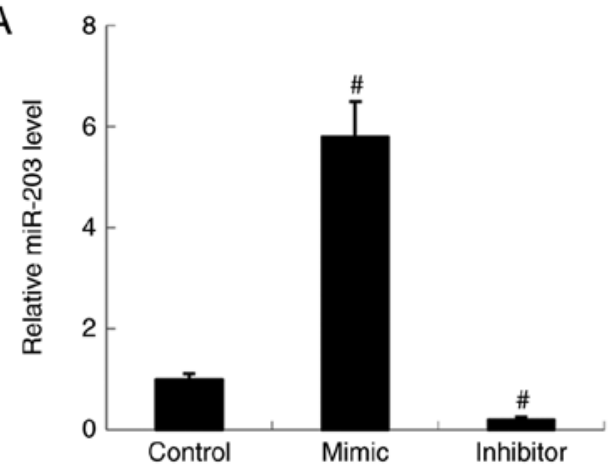

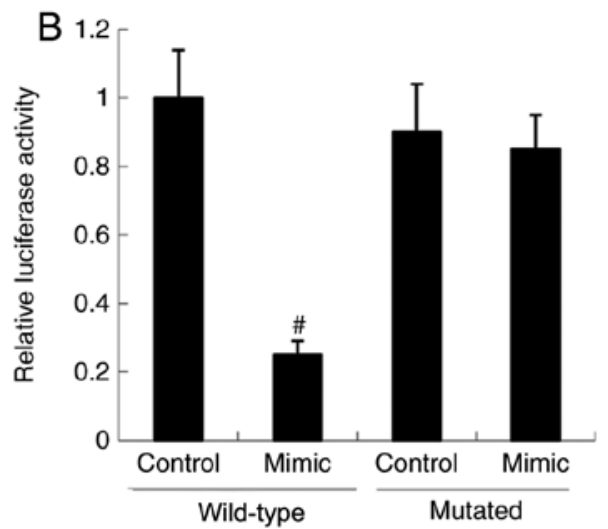
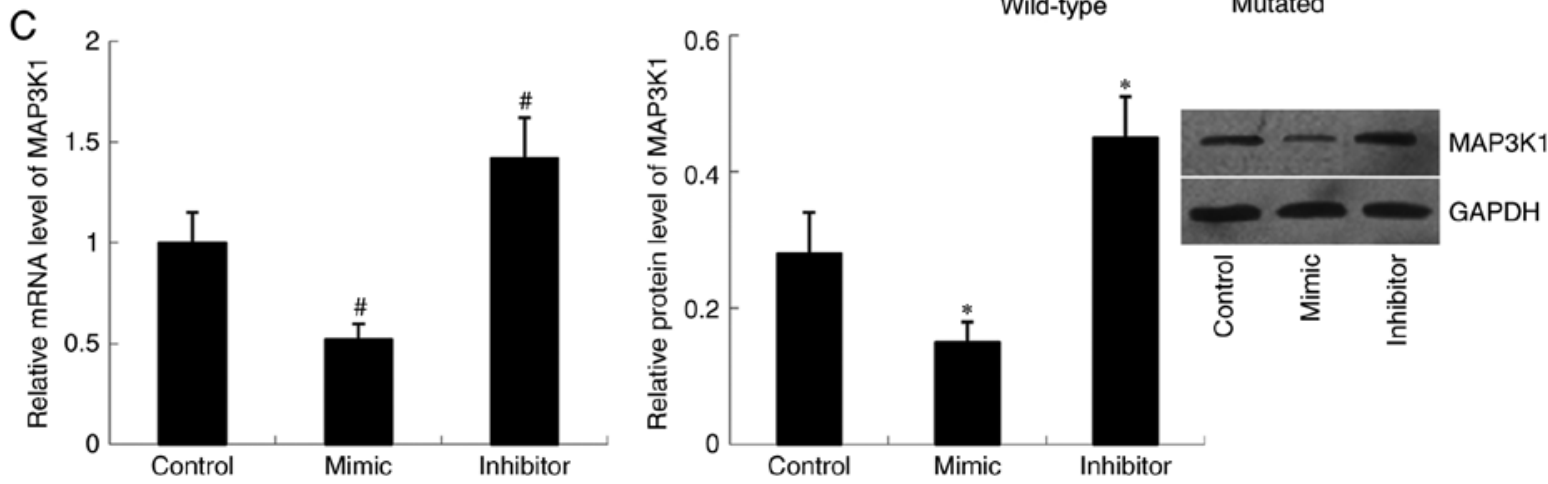

Figure 1. MAP3K1 is directly regulated by miR-203. (A) miR-203 expression in TE-1 cells following transfection with miR-203 mimic and miR-203 inhibitor. Data are analyzed using one-way analysis of variance, followed by Student-Keuls-Neuman test. (B) Relative luciferase activity of luciferase-expressing plasmids containing wild-type MAP3K1-3'UTR or mutant MAP3K1-3'UTR. Data are analyzed using unpaired two-tailed Student's t-test. (C) MAP3K1 mRNA and protein expression levels in TE-1 cells following transfection with miR-203 mimic and miR-203 inhibitor. Data are analyzed using one-way analysis of variance, followed by Student-Keuls-Neuman test. ${ }^{~} \mathrm{P}<0.05,{ }^{\#} \mathrm{P}<0.01$ vs. control (miR-negative control). MAP3K1, Mitogen-Activated Protein Kinase Kinase Kinase 1; miR, microRNA; UTR, untranslated region.

cells, cells were divided into the following three groups: Control group, MAP3K1 pcDNA3.1 group and MAP3K1 siRNA group; control, mimic and mimic + MAP3K1 groups, and control, inhibitor and inhibitor+MAP3K1 siRNA groups, as previously described. The activities of caspase 8 and caspase 3 in TE-1 cells were examined using the Caspase 8/Caspase 3 Colorimetric Assay kit (Nanjing KeyGen Biotech Co., Ltd.), according to the manufacturer's protocol.

Cell invasion assay. In order to investigate cell invasion ability of MAP3K1-overexpressed or MAP3K1-knockdown cells, cells were divided into the following groups: Control group, MAP3K1 pcDNA3.1 group and MAP3K1 siRNA group; control, mimic and mimic + MAP3K1 groups, and control, inhibitor and inhibitor + MAP3K1 siRNA groups, as aforementioned. The cell invasion assay was performed using Transwell inserts (Corning, Inc.) coated with Matrigel matrix (BD Biosciences) at $37^{\circ} \mathrm{C}$ for $30 \mathrm{~min}$. Cell suspension was prepared in serum-free medium at a density of $5 \times 10^{4}$ cells $/ \mathrm{ml}$ and added to the upper chambers. A total of $1 \mathrm{ml}$ of cell medium containing $10 \%$ FBS was added to the lower chambers. Following overnight incubation at $37^{\circ} \mathrm{C}$, cotton swabs were used to gently remove non-invaded cells. Cells on the lower surface of the membrane were fixed in $95 \%$ ethanol for $20 \mathrm{~min}$ and subsequently stained with hematoxylin for $10 \mathrm{~min}$ at room temperature. The number of invaded cells was counted under an inverted light microscope (Nikon Corporation; magnification, $\mathrm{x} 400$ ).
Statistical analysis. Statistical analysis was performed using SPSS software (version 19.0; SPSS, Inc.). Data are presented as the mean \pm standard deviation. Unpaired two-tailed Student's t-test and one-way analysis of variance, followed by Student-Keuls-Neuman test, were used to analyze the statistical difference between the respective groups. $\mathrm{P}<0.05$ was considered to indicate a statistically significant difference.

\section{Results}

MAP3K1 is directly regulated by $m i R-203$. miR-203 mimic and miR-203 inhibitor were transfected into cells, respectively. Relative miR-203 level was significantly increased in the mimic group $(\mathrm{P}<0.01)$ but decreased in the inhibitor group $(\mathrm{P}<0.01)$ compared with the control group (Fig. 1A). Subsequently, MAP3K1 expression was examined in the cells following transfection with miR-203 mimic and miR-203 inhibitor. RT-qPCR and western blot analyses demonstrated that transfection with miR-203 mimic decreased both MAP3K1 mRNA $(\mathrm{P}<0.01)$ and protein $(\mathrm{P}<0.05)$ expression compared with the control group. Conversely, relative MAP3K1 mRNA $(\mathrm{P}<0.05)$ and protein $(\mathrm{P}<0.01)$ levels were significantly increased in cells transfected with miR-203 inhibitor compared with the control group (Fig. 1C).

Luciferase-expressing plasmids containing the wild-type MAP3K1-3'UTR or mutant MAP3K1-3'UTR were constructed and co-transfected with miR-203 mimic or control. The results indicated that translational activity of the wild-type 
A

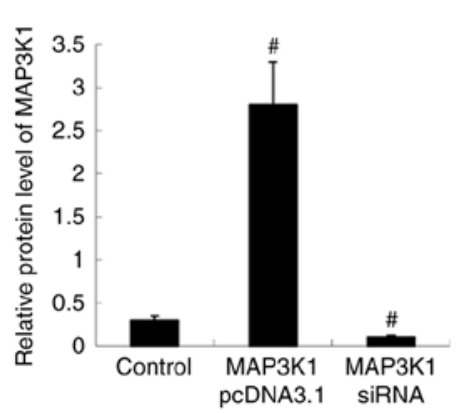

$\mathrm{B}$

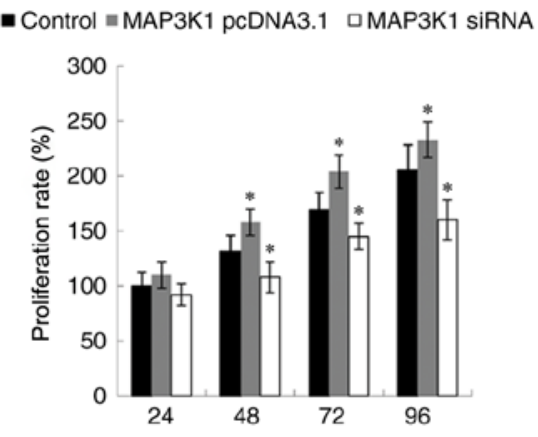

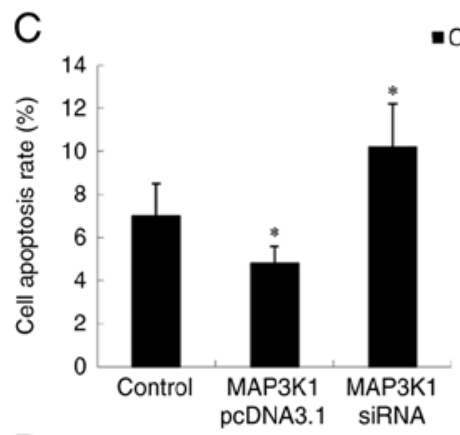
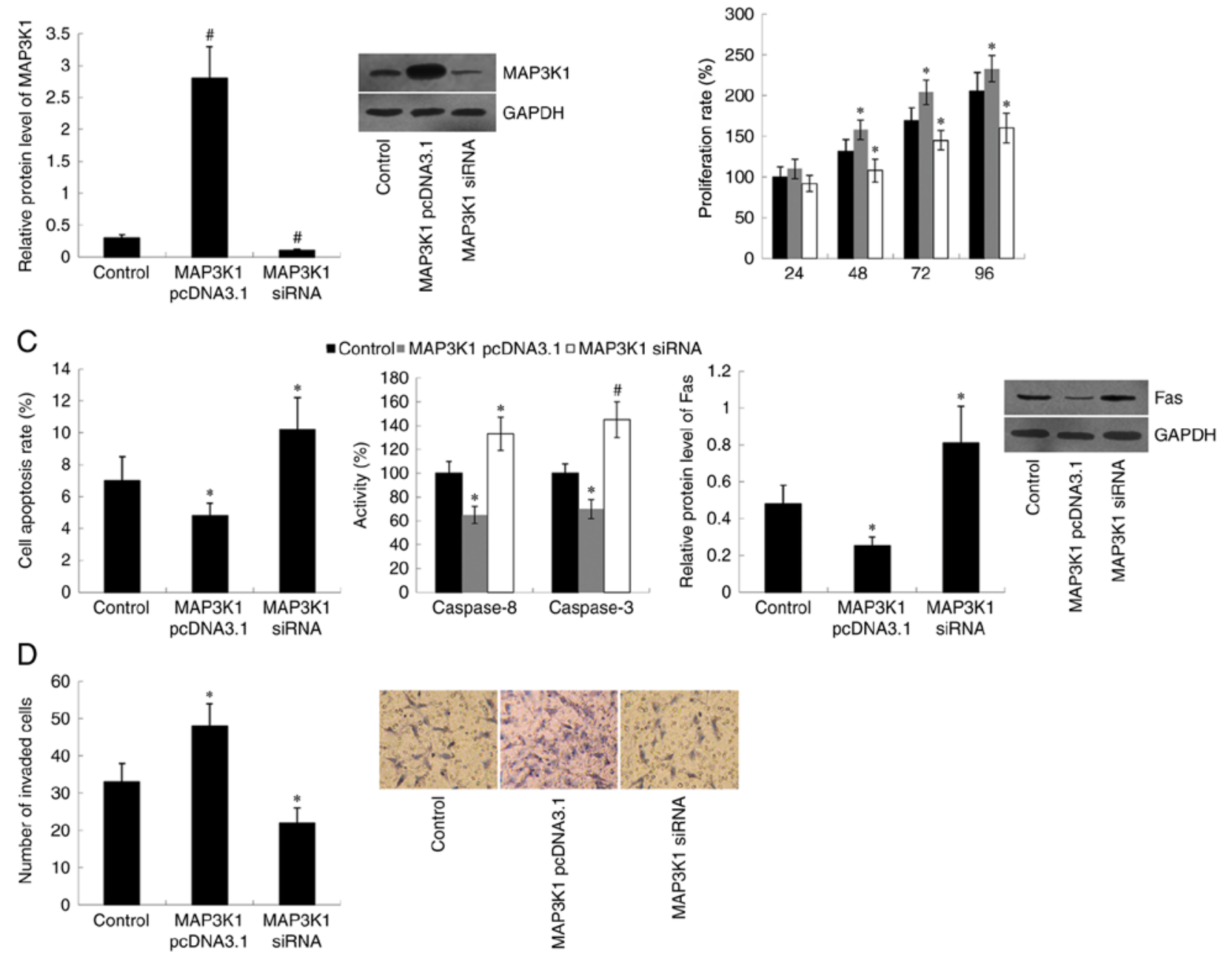

Figure 2. Effect of MAP3K1 on cell proliferation, apoptosis and invasion. (A) MAP3K1 protein expression in TE-1 cells following transfection with MAP3K1 pcDNA3.1 and MAP3K1 siRNA. Effect of MAP3K1 on cell (B) proliferation, (C) apoptosis and (D) invasion. Data are analyzed using one-way analysis of variance, followed by Student-Keuls-Neuman test. ${ }^{\prime} \mathrm{P}<0.05,{ }^{\text {}} \mathrm{P}<0.01$ vs. control (empty vector + scramble siRNA). MAP3K1, Mitogen-Activated Protein Kinase Kinase Kinase 1; si, small interfering.

MAP3K1-3'UTR was significantly decreased in the miR-203 mimic group compared with that of the control group $(\mathrm{P}<0.01)$. However, no significant difference in translational activity of the mutant MAP3K1-3'UTR was observed between the miR-203 mimic group and the control group (Fig. 1B).

Effect of MAP3K1 on cell proliferation, apoptosis and invasion. TE-1 cells were transfected with MAP3K1 pcDNA3.1 or MAP3K1 siRNA to overexpress or knockdown MAP3K1, respectively. The western blot analysis demonstrated that MAP3K1 protein expression was significantly upregulated in MAP3K1 pcDNA3.1-transfected cells $(\mathrm{P}<0.01)$ and downregulated in MAP3K1 siRNA-transfected cells $(\mathrm{P}<0.01)$, compared with the control group (Fig. 2A). Subsequently, the effect of MAP3K1 on cell proliferation, apoptosis and invasion was assessed. Results of the MTT assay indicated that transfection with MAP3K1 pcDNA3.1 significantly induced cell proliferation ( $\mathrm{P}<0.05$ at 48,72 and $96 \mathrm{~h}$ ), whereas transfection with MAP3K1 siRNA significantly inhibited cell proliferation $(\mathrm{P}<0.05$ at 48,72 and $96 \mathrm{~h}$ ) compared with the control group (Fig. 2B). Furthermore, cell apoptotic rate, Caspase $8 / 3$ activity and Fas protein expression were significantly decreased in MAP3K1 pcDNA3.1-transfected cells $(\mathrm{P}<0.05)$ and increased in MAP3K1 siRNA-transfected cells $(\mathrm{P}<0.05$ for cell apoptotic rate and Fas protein expression; $\mathrm{P}<0.01$ for Caspase $8 / 3$ activity) compared with the control group (Fig. 2C). MAP3K1 failed to demonstrate effects on cell proliferation at $24 \mathrm{~h}$ (Fig. 2B) following transfection with MAP3K1 pcDNA3.1 or MAP3K1 siRNA, thus cell invasion ability was assessed within $24 \mathrm{~h}$ (overnight, 12-16 h). Therefore, the change in cell invasion ability could not be affected by cell proliferation in the study. The results demonstrated that MAP3K1 overexpression promoted cell invasion $(\mathrm{P}<0.05)$, while MAP3K1 knockdown inhibited cell invasion $(\mathrm{P}<0.05)$ compared with the control group (Fig. 2D).

MAP3K1 reverses the effect of miR-203 on cell proliferation, apoptosis and invasion. Cells were co-transfected with miR-203 mimic and MAP3K1 pcDNA3.1, and RT-qPCR analysis was performed to detect miR-203 expression. As presented in Fig. 3A, overexpression of MAP3K1 did not affect the increased miR-203 expression exhibited in 
A
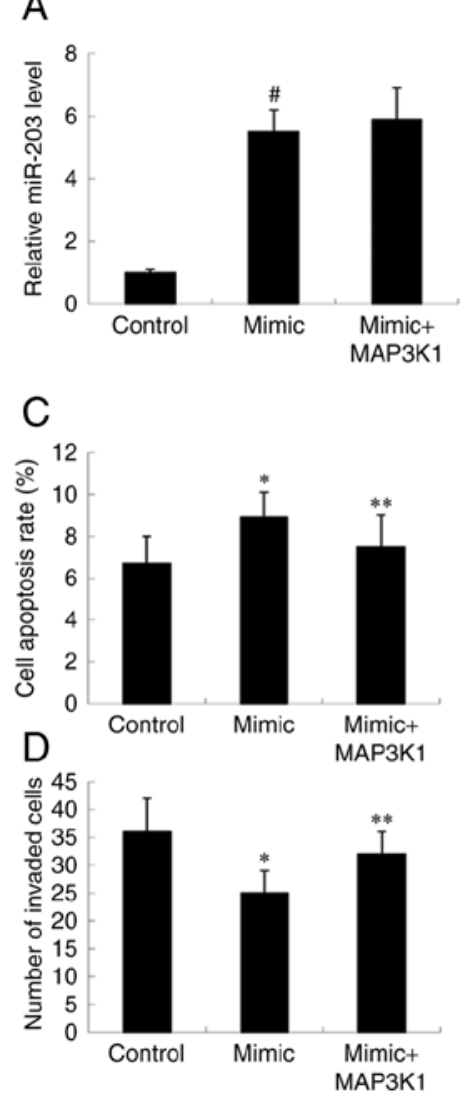
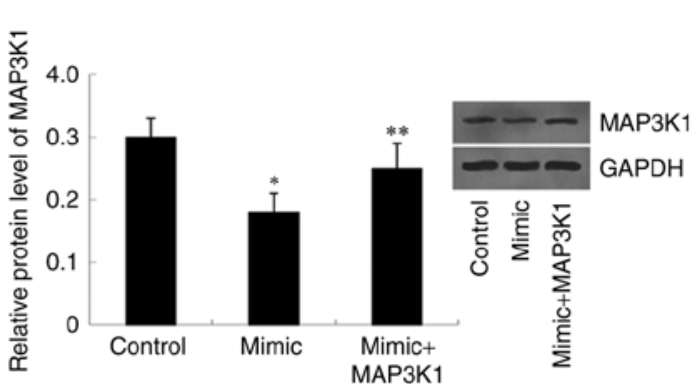

$\mathrm{B}$

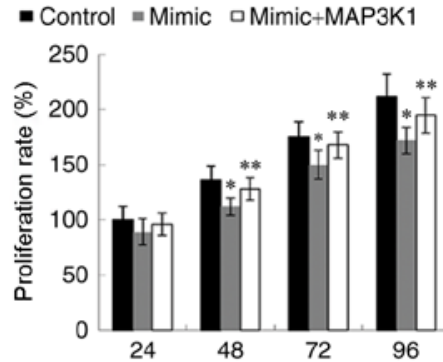

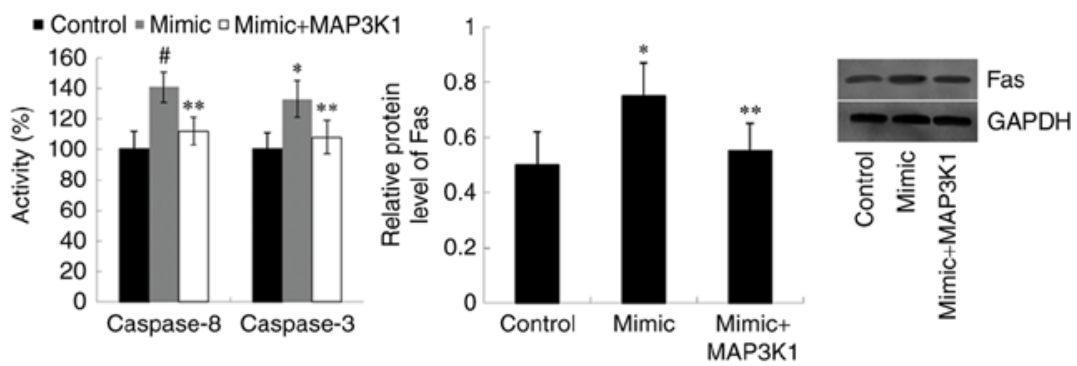

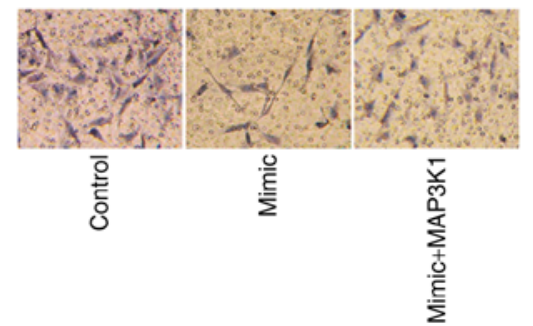

Figure 3. Overexpression of MAP3K1 reverses the effect of miR-203 mimic on cell proliferation, apoptosis and invasion. (A) miR-203 and MAP3K1 expression in TE-1 cells following transfection with miR-203 mimic and the MAP3K1 overexpression plasmid. Overexpression of MAP3K1 reversed the (B) inhibitory effect of miR-203 mimic on cell proliferation, (C) promotive effect of miR-203 mimic on cell apoptosis and (D) inhibitory effect of miR-203 mimic on cell invasion. Data are analyzed using one-way analysis of variance, followed by Student-Keuls-Neuman test. ${ }^{*} \mathrm{P}<0.05,{ }^{\#} \mathrm{P}<0.01$ vs. control (miR-negative control + empty vector); ${ }^{* *} \mathrm{P}<0.05$ vs. miR-203 mimic group. MAP3K1, Mitogen-Activated Protein Kinase Kinase Kinase 1; miR, microRNA.

miR-203 mimic-transfected cells. However, western blot analysis demonstrated that the decreased MAP3K1 protein expression observed in miR-203 mimic-transfected cells was significantly rescued following transfection with MAP3K1 pcDNA3.1 $(\mathrm{P}<0.05)$. Furthermore, transfection with miR-203 mimic suppressed the cell proliferation rate compared with the control group $(\mathrm{P}<0.05$ at 48, 72 and 96 h). However, overexpression of MAP3K1 reversed the inhibitory effect of miR-203 mimic on cell proliferation. Cells co-transfected with miR-203 mimic and the MAP3K1 overexpression plasmid proliferated at a faster rate compared with the miR-203 mimic-transfected cells ( $\mathrm{P}<0.05$ at 48,72 and 96 h) (Fig. 3B). As presented in Fig. 3C, the apoptotic rate, Caspase $8 / 3$ activity and Fas protein expression increased in cells transfected with miR-203 mimic compared with the control group $(\mathrm{P}<0.05$ for cell apoptotic rate and Fas protein expression; $\mathrm{P}<0.01$ for Caspase $8 / 3$ activity). However, the increased cell apoptotic rate observed in miR-203 mimic-transfected cells was significantly reversed following co-transfection with the MAP3K1 overexpression plasmid $(\mathrm{P}<0.05)$. Furthermore, overexpression of MAP3K1 was demonstrated to reverse the inhibitory effect of miR-203 mimic on cell invasion $(\mathrm{P}<0.05)$ (Fig. 3D).

miR-203 and MAP3K1 expression in cells co-transfected with miR-203 inhibitor and MAP3K1 siRNA was subsequently assessed. MAP3K1 siRNA significantly decreased MAP3K1 protein expression in cells transfected with miR-203 inhibitor
$(\mathrm{P}<0.05)$; however, MAP3K1 siRNA was demonstrated to have no effect on miR-203 expression compared with the control group (Fig. 4A). As expected, the effect of miR-203 inhibitor on cell proliferation, apoptosis and invasion was weakened by MAP3K1 siRNA $(\mathrm{P}<0.05)$ (Fig. 4B-D).

\section{Discussion}

miRNA is known to regulate several target genes (18). Previous studies have reported a number of target genes of miR-203, including twist 1, RGS17, PBOV1, EGR1 and FGF2 $(13,12,19,20)$. Using prediction software, the present study hypothesized that MAP3K1 may be a target gene of miR-203. Results of the luciferase reporter assay demonstrated that miR-203 directly binds to the 3'UTR of MAP3K1, while western blotting analysis indicated that MAP3K1 protein expression was upregulated by miR-203.

MAP3K1 is a serine/threonine kinase belonging to the MAP3K family $(21,22)$. It is activated by various stimuli, such as cellular stress, growth factors and cytokines, and is part of several signal transduction cascades, including the ERK, JNK and NF- $\kappa$ B signaling pathways (23). Previous studies have demonstrated that MAP3K1 plays critical roles in multiple aspects of cell physiology $(24,25)$, including cell proliferation, apoptosis and motility in both normal and tumor cells $(22,26)$. For example, MAP3K1-targeting artificial 
A
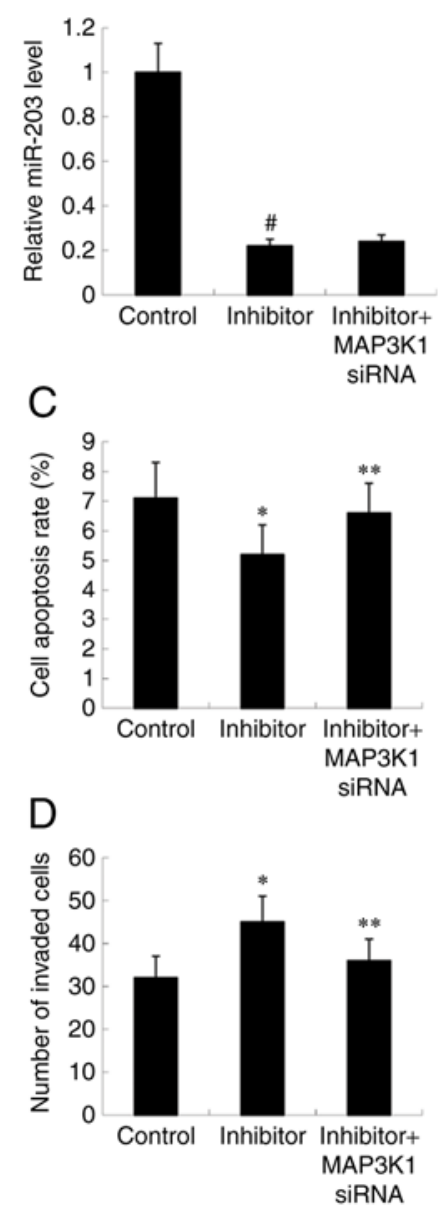

B

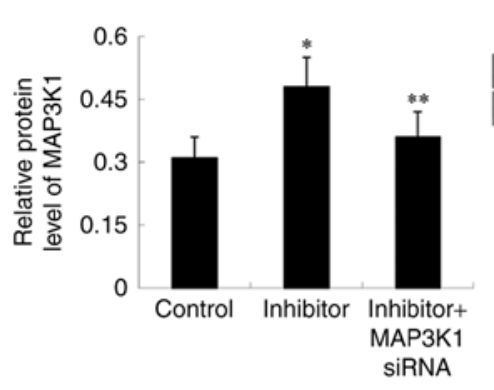

- Control = Inhibitor a Inhibitor+MAP3K1 siRNA

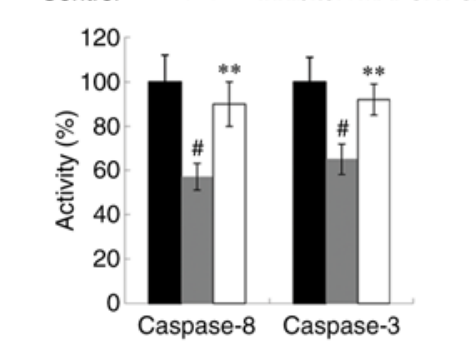

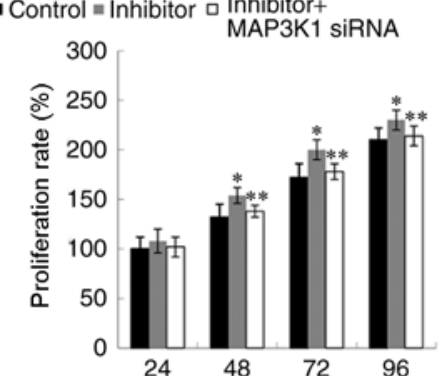
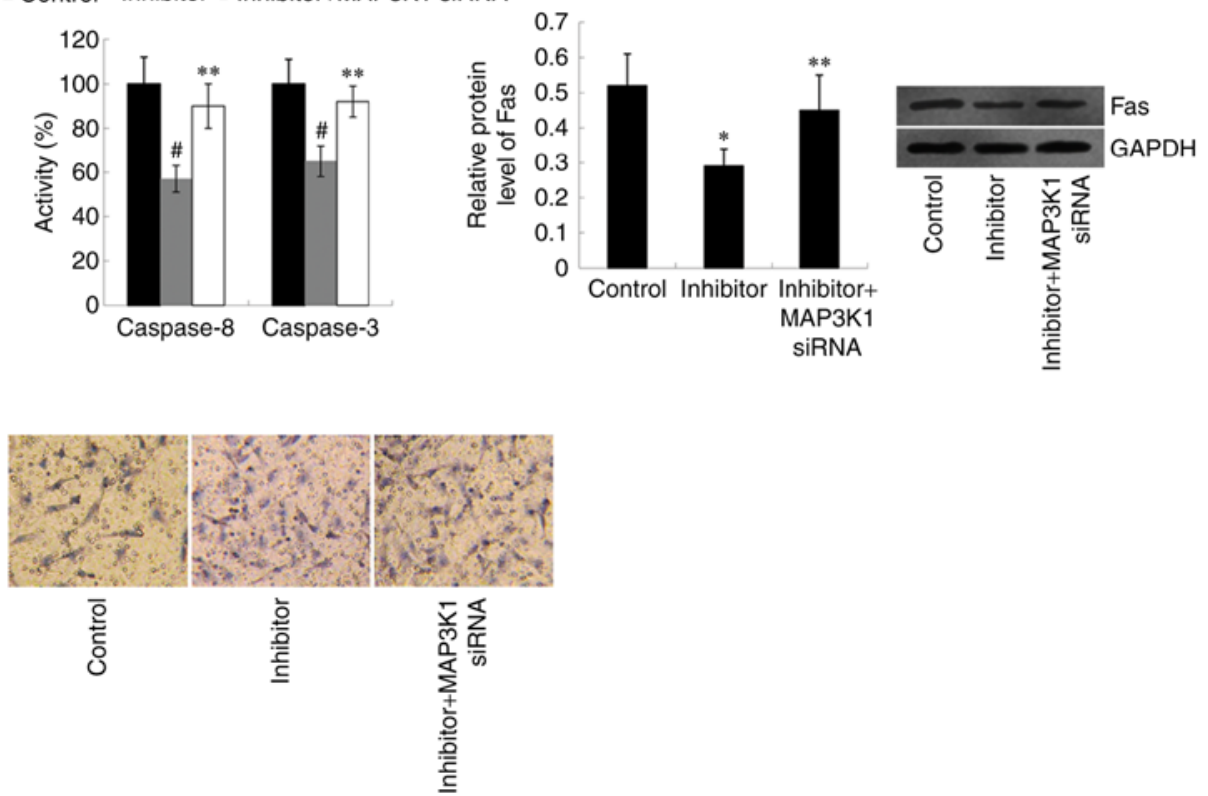

Figure 4. MAP3K1 knockdown reverses the effect of miR-203 inhibitor on cell proliferation, apoptosis and invasion. (A) miR-203 and MAP3K1 expression in TE-1 cells following transfection with miR-203 inhibitor and MAP3K1 siRNA. MAP3K1 knockdown reversed the (B) promotive effect of miR-203 inhibitor on cell proliferation, (C) inhibitory effect of miR-203 inhibitor on cell apoptosis and (D) promotive effect of miR-203 inhibitor on cell invasion. Data are analyzed using one-way analysis of variance, followed by Student-Keuls-Neuman test. ${ }^{*} \mathrm{P}<0.05$, ${ }^{*} \mathrm{P}<0.01 \mathrm{vs}$. control (miR-negative control + scramble siRNA); ${ }^{* *} \mathrm{P}<0.05$ vs. inhibitor group. MAP3K1, Mitogen-Activated Protein Kinase Kinase Kinase 1; miR, microRNA; si, small interfering.

miRNA suppresses the growth and invasion of breast cancer both in vivo and in vitro (27). Furthermore, depletion of MAP3K1 inhibits survival, invasion and migration of human pancreatic cancer cells $(28,29)$. Bian et al $(30)$ reported that MAP3K1 activity is essential for Lysophosphatidic acid-stimulated ovarian cancer cell migration (30). Taken together, these findings indicate that MAP3K1 plays a facilitating role in the development of several types of tumor. However, the role of MAP3K1 in esophageal cancer remains to be elucidated.

The present study performed in vitro gain-of-function and loss-of-function experiments, in order to investigate the effect of MAP3K1 on TE-1 cell proliferation, apoptosis and invasion. The results demonstrated that MAP3K1 promoted cell proliferation and invasion, and inhibited cell apoptosis, thus exerting a tumor-promoting role in esophageal cancer. These results are consistent with those of a previous study, which reported that MAP3K1 acts as a tumor promoter in EC9706 cells (31).

Furthermore, MAP3K1 overexpression plasmid or MAP3K1 siRNA were transfected into TE-1 cells in the presence of miR-203 mimic or miR-203 inhibitor, in order to determine whether MAP3K1 was involved in the varying effects of miR-203 on cell proliferation, apoptosis and invasion. It was demonstrated that overexpression of MAP3K1 reversed the suppressed cell proliferation and invasion abilities induced by miR-203 mimic, and the inhibitory effect of miR-203 mimic on cell apoptosis. Furthermore, MAP3K1 siRNA weakened the effect of miR-203 inhibitor on cell proliferation, apoptosis and invasion. Theses results suggest that MAP3K1 plays a notable role in mediating the effect of miR-203 on esophageal cancer cell proliferation, apoptosis and invasion.

The present study demonstrated that the cell apoptosis rates were $<10 \%$. Regarding flow cytometry analysis, cell debris existed, which may have affected accurate measurement of the cell apoptotic rate. A limitation of the present study is that cell proliferation was only detected via an MTT assay. Future studies will aim to include cell cycle analysis to accurately investigate cell proliferation.

Taken together, the results of the present study provide a novel molecular mechanism underlying the role of miR-203 in esophageal cancer, and suggest that MAP3K1 is a direct target of miR-203. miR-203 was able to suppress esophageal cancer 
cell proliferation and invasion, and induce cell apoptosis at least partially by inhibiting MAP3K1 expression.

\section{Acknowledgements}

Not applicable.

\section{Funding}

No funding was received.

\section{Availability of data and materials}

The datasets used and/or analyzed during the present study are available from the corresponding author upon reasonable request.

\section{Authors' contributions}

WY and MZ designed the present study. MZ, WF, LW and XY performed the experiments and analyzed the data. MZ drafted the initial manuscript. All authors have read and approved the final manuscript.

\section{Ethics approval and consent to participate}

Not applicable.

\section{Patient consent for publication}

Not applicable.

\section{Competing interests}

The authors declare that they have no competing interests.

\section{References}

1. Global Burden of Disease Cancer Collaboration; Fitzmaurice C, Dicker D, Pain A, Hamavid H, Moradi-Lakeh M, MacIntyre MF Allen C, Hansen G, Woodbrook R, et al: The global burden of cancer 2013. JAMA Oncol 1: 505-527, 2015.

2. Yokota T, Igaki H, Kato K, Tsubosa Y, Mizusawa J, Katayama H, Nakamura K, Fukuda H and Kitagawa Y: Accuracy of preoperative diagnosis of lymph node metastasis for thoracic esophageal cancer patients from JCOG9907 trial. Int J Clin Oncol 21: 283-288, 2016

3. Kamangar F, Dores GM and Anderson WF: Patterns of cancer incidence, mortality, and prevalence across five continents: Defining priorities to reduce cancer disparities in different geographic regions of the world. J Clin Oncol 24: 2137-2150, 2006.

4. Parkin DM, Bray F, Ferlay J and Pisani P: Global cancer statistics, 2002. CA Cancer J Clin 55: 74-108, 2005.

5. Mohr AM and Mott JL: Overview of microRNA biology. Semin Liver Dis 35: 3-11, 2015.

6. Vienberg S, Geiger J, Madsen S and Dalgaard LT: MicroRNAs in metabolism. Acta Physiol (Oxf) 219: 346-361, 2017.

7. Reddy KB: MicroRNA (miRNA) in cancer. Cancer Cell Int 15: 38,2015 .

8. Iorio MV and Croce CM: MicroRNA dysregulation in cancer: Diagnostics, monitoring and therapeutics. A comprehensive review. EMBO Mol Med 4: 143-159, 2012.

9. Kiselev FL: MicroRNA and cancer. Mol Biol (Mosk) 48 : 232-242, 2014 (In Russian).

10. Mei LL, Qiu YT,Zhang B and Shi ZZ: MicroRNAs in esophageal squamous cell carcinoma: Potential biomarkers and therapeutic targets. Cancer Biomark 19: 1-9, 2017.
11. Liang Y, Yang W, Zhu Y and Yuan Y: Prognostic role of microRNA-203 in various carcinomas: Evidence from a meta-analysis involving 13 studies. SpringerPlus 5: 1538, 2016.

12. Chi Y, Jin Q, Liu X, Xu L, He X, Shen Y, Zhou Q, Zhang J and Jin M: miR-203 inhibits cell proliferation, invasion, and migration of non-small-cell lung cancer by downregulating RGS17. Cancer Sci 108: 2366-2372, 2017.

13. Shen J, Zhang J, Xiao M, Yang J and Zhang N: miR-203 suppresses bladder cancer cell growth and targets twist1. Oncol Res 26: 1155-1165, 2018.

14. Chen LZ, Ding Z, Zhang Y, He ST and Wang XH: MiR-203 over-expression promotes prostate cancer cell apoptosis and reduces ADM resistance. Eur Rev Med Pharmacol Sci 22: 3734-3741, 2018.

15. Hezova R, Kovarikova A, Srovnal J, Zemanova M, Harustiak T, Ehrmann J, Hajduch M, Svoboda M, Sachlova M and Slaby O: Diagnostic and prognostic potential of miR-21, miR-29c, miR-148 and miR-203 in adenocarcinoma and squamous cell carcinoma of esophagus. Diagn Pathol 10: 42, 2015.

16. Zhang F, Yang Z, Cao M, Xu Y, Li J, Chen X, Gao Z, Xin J, Zhou S, Zhou Z, et al: MiR-203 suppresses tumor growth and invasion and down-regulates MiR-21 expression through repressing Ran in esophageal cancer. Cancer Lett 342: 121-129, 2014.

17. Livak KJ and Schmittgen TD: Analysis of relative gene expression data using real-time quantitative PCR and the 2(-Delta Delta $\mathrm{C}(\mathrm{T})$ ) method. Methods 25: 402-408, 2001.

18. Bartel DP: MicroRNAs: Genomics, biogenesis, mechanism, and function. Cell 116: 281-297, 2004.

19. Zhang SY, Gao F, Peng CG, Zheng CJ and Wu MF: Hsa-miR-203 inhibits fracture healing via targeting PBOV1. Eur Rev Med Pharmacol Sci 22: 5797-5803, 2018.

20. Shi K, Qiu X, Zheng W, Yan D and Peng W: MiR-203 regulates keloid fibroblast proliferation, invasion, and extracellular matrix expression by targeting EGR1 and FGF2. Biomed Pharmacother 108: 1282-1288, 2018.

21. Pham TT, Angus SP and Johnson GL: MAP3K1: Genomic alterations in cancer and function in promoting cell survival or apoptosis. Genes Cancer 4: 419-426, 2013.

22. Hagemann C and Blank JL: The ups and downs of MEK kinase interactions. Cell Signal 13: 863-875, 2001.

23. Bonvin C, Guillon A, van Bemmelen MX, Gerwins $P$, Johnson GL and Widmann C: Role of the amino-terminal domains of MEKKs in the activation of NF kappa B and MAPK pathways and in the regulation of cell proliferation and apoptosis. Cell Signal 14: 123-131, 2002.

24. Kyriakis JM and Avruch J: Mammalian mitogen-activated protein kinase signal transduction pathways activated by stress and inflammation. Physiol Rev 81: 807-869, 2001.

25. Uhlik MT, Abell AN, Cuevas BD, Nakamura K and Johnson GL: Wiring diagrams of MAPK regulation by MEKK1, 2, and 3. Biochem Cell Biol 82: 658-663, 2004.

26. Lange-Carter CA, Pleiman CM, Gardner AM, Blumer KJ and Johnson GL: A divergence in the MAP kinase regulatory network defined by MEK kinase and Raf. Science 260: 315-319, 1993.

27. Liu C, Wang S, Zhu S, Wang H, Gu J, Gui Z, Jing J, Hou X and Shao Y: MAP3K1-targeting therapeutic artificial miRNA suppresses the growth and invasion of breast cancer in vivo and in vitro. SpringerPlus 5: 11, 2016.

28. Hirano T, Shino Y, Saito T, Komoda F, Okutomi Y, Takeda A Ishihara T, Yamaguchi T, Saisho H and Shirasawa H: Dominant negative MEKK1 inhibits survival of pancreatic cancer cells. Oncogene 21: 5923-5928, 2002.

29. Su F, Li H, Yan C, Jia B, Zhang Y and Chen X: Depleting MEKK1 expression inhibits the ability of invasion and migration of human pancreatic cancer cells. J Cancer Res Clin Oncol 135: 1655-1663, 2009.

30. Bian D, Su S, Mahanivong C, Cheng RK, Han Q, Pan ZK, Sun P and Huang S: Lysophosphatidic acid stimulates ovarian cancer cell migration via a ras-MEK kinase 1 pathway. Cancer Res 64: 4209-4217, 2004.

31. Zang WQ, Yang X, Wang T, Wang YY, Du YW, Chen XN, Li M and Zhao GQ: MiR-451 inhibits proliferation of esophageal carcinoma cell line EC9706 by targeting CDKN2D and MAP3K1. World J Gastroenterol 21: 5867-5876, 2015.

This work is licensed under a Creative Commons Attribution-NonCommercial-NoDerivatives 4.0 International (CC BY-NC-ND 4.0) License. 